\title{
Perception of healthy and unhealthy food among Chinese adolescents
}

\author{
Kara Chan* \\ Professor, Department of Communication Studies \\ Hong Kong Baptist University \\ karachan@hkbu.edu.hk \\ 85234117836 \\ Dr. Tommy Tse, Assistant Professor \\ Department of Sociology \\ The University of Hong Kong \\ tommyt@hku.hk \\ 85239178532 \\ Dr. Daisy Tam, Research Assistant Professor \\ Department of Humanities and Creative Writing \\ Hong Kong Baptist University \\ daisytam@hkbu.edu.hk \\ 85234115871 \\ Anqi Huang \\ Research Assistant \\ Department of Communication Studies \\ Hong Kong Baptist University \\ angelinhk@gmail.com \\ 85266774353 \\ Manuscript accepted for publication \\ Young Consumers
}

Acknowledgement: This study was supported by a General Research Fund from the Research Grants Council (Project No. 240713).

April 13, 2016

*corresponding author 


\title{
Perception of healthy and unhealthy snacks among Chinese adolescents
}

\begin{abstract}
Purpose - The purpose of this paper is to explore snacking behavior and perspectives on healthy and unhealthy food choices among adolescents in mainland China.

Design/methodology/approach - Four focus-group interviews were conducted.

Altogether 24 participants were recruited in Changsha, a second-tier city in China, through a convenience sampling process. They were asked to report their snacking behaviors, identify whether certain snacks are healthy or unhealthy, and elaborate on factors affecting food choices.
\end{abstract}

Findings - Snacking was prevalent among the participants. The most frequently consumed snacks included fruit, milk, and instant noodles. Participants' evaluations for the healthiness of foods were based on the actual nutritional values of those foods, the effects on growth and body weight, and word-of-mouth. Choice of snack was driven mainly by taste, image, convenience, and health consciousness.

Research limitations/implications -The finding was based on a non-probability sample. The study also did not explore the contexts where snacks were consumed. Originality/value - This is the first study on snacking behaviors among adolescents conducted in a second-tier city in China using focus group methodology. 
Keywords - consumer perceptions; food marketing; qualitative method; diet;

adolescents

Paper type - Research paper 


\section{Perceptions of healthy and unhealthy snacks among Chinese adolescents}

\section{Introduction}

In the 30 years since China's economic reform, food markets in China have

diversified and prospered. With an average growth rate of 30 percent from 2006 to

2012, China has become the world's largest market for food and grocery retail (EU

SME Centre, 2013). China has endorsed a basic three meals a day culture, and

snacking was not prevalent until the last decade (Zhai et al., 2014). However, a

remarkable increase in snacking behavior has occurred since 2004 according to the

findings of the China Health and Nutrition Survey (CHNS) in 1991, 2004, 2006 and

2009 (Wang et al., 2012). The China Health and Nutrition Survey (CHNS) was

introduced in 1989 and was conducted every two to three years from 1991 to 2011.

The 2011 edition covered nine provinces with a sample size of 5,884 households and

27,447 individuals (Zhang et al, 2014). Nearly half of the child and adolescent

respondents to the 2009 survey snacked, and over one third of the adult respondents

consumed snacks. More recently, a survey of parents in Shanghai found that children

often consumed snacks with high caloric values and fat contents, such as cakes,

cookies, and chips. Altogether seventy percent of Chinese parents perceived that

snacks were able to provide nutrition for their children (Chen, 2014). Although the

prevalence of snacking and the energy intake from snacks among Chinese were lower 
than those of their U.S. counterparts, snacks have become an indispensable part of the Chinese diet (Wang et al., 2012). A visit to an upscale supermarket in Beijing revealed that items such as salty snacks, chocolate, and cakes took up a considerable portion of shelf space. Chinese teenagers, with their intense schedules of educational activities, often eat meals and snacks away from home. As a result, retail outlets and peers compete with parents for influence on food choice (Veeck et al., 2014). Young consumers' attitudes and tastes for food are influenced by the socializing agents including family and friends (Young, 2003). As with developed economies, the guiding principles for food choices among Chinese adolescents include food safety, taste, nutrition, price, and (for girls) weight control (Veeck et al., 2014). However, food culture among Chinese adolescents differs from their western counterparts in two aspects. First, Chinese adolescents often share meals and snacks. One study found that children often learn about new snacks from classmates through food sharing (Guo, 2000). Because of the country's traditionally collective culture, children's food consumption is also influenced by extended family members, including parents and grandparents. For example, one study showed that school-aged children cared for by grandparents were more likely to consume more sugar-added drinks and unhealthy snacks (Li et al., 2015). Furthermore, it was found that the majority of meal decisions were made close to the students' schools (Veeck et al., 2014). This can be attributed to 
the long hours students spend at school, in particular hours spent after classes in preparation for public examinations.

Adolescence is a critical period for both physical and mental growth, as the eating habits and nutritional intake of adolescents have long-term influence on their health (U.S. Department of Health and Human Services, 1988). During this stage, parental influence on food choice begins to give way to adolescents' increased autonomy in food consumption (Fitzgerald et al., 2010). The population in the age group of 10 to 19 is nearly 180 million in China, compared to 45 million in the US (The World Factbook, 2015), making members of this age group important targets for the food and beverage market. Due to urbanization and increasing household incomes, adolescents in urban China are exposed to abundant food marketing communications, the growing presence of fast-food chains, and increasing choices of packaged snacks. As of 2014, fast-food restaurant chains in China were dominated by three brands, including KFC (around 4,600 outlets), Dicos (around 2,200 outlets), and McDonald's (around 2,000 outlets) (Sun, 2014). Major marketers of packaged foods and beverages, including Nestles, Coca-Cola, Want Want, and Kraft, have promoted their products actively in China (The Economist, 2012). A content analysis of free-to-air television in three Chinese provinces found that the average number of food advertisements ranged from 5.3 per hour in Heilongjiang to 8.3 per hour in Shanghai. The most 
frequently advertised food products were sugar-sweetened drinks, savory snack foods with added salt or fat, and milk and yoghurts (Kelly et al., 2014). Similarly, Powell et al. (2007) found that the majority of food advertisements seen on television by American children and adolescents were for foods of low nutritional content.

\section{Youth and snacking behavior}

What is snacking? It is typically defined as a distinctive eating behavior outside the three main meals, or else the consumption of small-portion food eaten with fingers rather than cutlery, that requires relatively little time or knowledge to make, and tends to be more individualistic and casual than a proper meal (Marshall, 2010; Wang et al., 2008). Snacking and snack foods are often associated with fun, freedom, and excitement (Marshall, 2010). Parents and teachers sometimes used snacks as rewards for children, and the withholding of snacks as punishments, making snacks even more desirable (Kline, 2011). The types of food and drinks usually classified as snacks for children consist of bread, biscuits, carbonated soft drinks, confectionery, crisps, desserts, ice cream, fruit, sugary breakfast cereals, fast food savory snacks, and other processed foods (Marshall, 2010; Yu et al., 2008). Snacks can also be categorized as “morning snacks, afternoon snacks and evening snacks" (Wang et al., 2012, p. 255).

Snacking among children and adults in China occurs most often in the evening, followed by snacking in the afternoon (Wang et al., 2008). Compared to adolescents 
from developed nations such as the United States, snacking in China is a relatively uncommon occurrence; as one study showed, altogether 91 percent of U.S. children snacked, from which they obtained a large amount ( 24 percent) of their daily calories, whereas only 11 percent of Chinese children snacked regularly, from which they obtained a smaller proportion (8 percent) of their total calories (Waller et al., 2003). Despite this disparity, however, snacks have nonetheless become an indispensable part of Chinese diet (Wang et al., 2012).

A survey of adolescents ages 12 to 14 found that higher socioeconomic status and urban residency correlated positively with consumption of fruit and high energy food. More than 70 percent of respondents expressed preference of Western foods such as soft drinks and chocolates (Shi et al., 2005). Teenage informants commented that they should avoid eating packaged snacks because these foods were expensive and unhealthy. However, many of them reported that they often shared snacks among friends in schools (Veech et al., 2014).

\section{Perception and practices of healthy eating among adolescents}

A study on adolescents in Hong Kong found that having a balanced diet, eating at regular times, and eating according to the food pyramid were considered healthy; also that eating a narrow range of foods, eating at irregular times, and eating food with preservatives or additives were considered unhealthy (Chan et al., 2009b). Another 
study found that healthy eating was perceived to involve moderation, balance, and variety (Croll et al., 2001). Stevenson et al. (2007) found that adolescents frequently linked healthy eating with dieting and considered healthy eating a temporary measure to prevent obesity. However, healthy eating was not considered important to teenagers as they believed they were not the target (Croll et al., 2001).

Adolescents in Hong Kong most often consumed unhealthy food at parties and social gatherings, as well as when eating out (Chan et al., 2009b). A focus-group study among adolescents ages 13 to 15 found that adolescents most often consumed healthy food at home. Homemade food tended to be healthier than commercial food because it was often prepared with less sugar, oil, and salt, and was often free of monosodium glutamate (MSG) (Chan et al., 2009a). However, awareness of healthy eating may not lead to healthy food choices in daily practice (Brown et al., 2000; Stevenson et al., 2007). Previous studies identified several barriers to healthy eating among adolescents, including preference for fast food due to cost consideration and taste, as well as poor school meal provision. Also, family support, making healthy food more accessible, and concern about physical appearance were identified as facilitators of healthy eating (Shepherd et al., 2015). According to a focus-group study among adolescents in the U.K., sensory properties (including taste, texture, 
appearance, and smell) were identified as the most important factor influencing food choice (Stevenson et al., 2007).

\section{Changes in food habits in China}

Changes in Chinese food habits were documented in The China Health and Nutrition Survey (CHNS) (Zhang et al, 2014). The major trends in food consumption analyzed and reported by various scholars were summarized as follows:

- A transition from diets dominated by coarse grains and carbohydrates to diets dominated by refined rice and wheat, as well as a significant drop in carbohydrate intake (Du et al., 2014);

- An increase in intake of animal-source foods (Du et al, 2014);

- A shift from healthy cooking methods of steaming, baking, and boiling to the unhealthy cooking method of frying (Zhai et al, 2014);

- An increase in snack consumption and eating away from home (Zhai et al, 2014).

\section{Snacking and obesity}

In view of the growing prevalence of snacking among adolescents, there is concern among parents and educators about the link between snacking behavior and obesity. Evidence that children's exposure to food advertising was positively correlated with their consumption of the advertised food brands as well as their consumption of 
energy-dense product categories was presented by Buijzen et al. (2008). A survey found that increased snacking was associated with increased daily energy, and the association was higher for older children than for younger children (Taillie et al., 2015). A review of studies conducted between 2000 and 2011 found that frequency of snacking was associated with higher intake of total energy as well as energy gained from sugar. However, a majority of these studies did not find evidence of a relationship between snacking behavior and weight status (Larson and Story, 2013).

\section{Research objectives}

Adolescents' snacking behavior and their perceptions of healthy and unhealthy food deserve study. Adolescents are becoming more and more autonomous in their food choice behavior (Veeck et al., 2014). However, their criteria for judging healthy and unhealthy foods are relatively unstudied. With the aim of generating meaningful and testable research hypotheses, the following research questions are posed:

RQ1. What is the snacking behavior of Chinese adolescents?

RQ2. How do Chinese adolescents identify healthy or unhealthy snacks?

RQ3. What are the major factors that influence the consumption of healthy or unhealthy food?

\section{Methodology}


This study employed an interpretivist approach (Neuman, 2003) using a qualitative methodology. It was an exploratory study designed to provide insight for future quantitative studies. A qualitative approach was adopted because we have little knowledge about how adolescents in Mainland China perceive healthy or unhealthy snacks. Focus-group sessions were adopted as the preferred method of enquiry (Silverman, 2005), since a group format can better accommodate individuals with different perspectives, as well as generating an understanding of the extent to which meanings, perceptions, and emotions associated with healthy food are negotiated, shared or divided. The focus group interviews were conducted in June 2014

\section{Location and sample}

A second-tier city, Changsha, was selected. Marketers classify cities in China into different tiers mainly based on economic development, provincial GDP, and advancement in infrastructure. China's first-tier cities are Beijing, Shanghai, Guangzhou and Shenzhen. Third-tier cities include medium-sized cities from various provinces (The American Chamber of Commerce in Shanghai, n.d.). Most consumer studies are conducted in first-tier cities in China. However, some second-tier cities are considered future powerhouses of economic growth due to their improved infrastructure and influx of investment and talents (Mullich, 2011). As an important city in central China, Changsha is economically strong with a per capita GDP of RMB 
89,900 in 2012 (HKTDC, 2014). The population of Changsha is over 6 million.

Changsha is often selected in social science research to represent an inland second-tier city (Yang et al., 2010).

A convenience sampling method was adopted. Through personal networks, 24

Chinese adolescents ages 12 to 13 studying in grade 1 of a public junior high school

(equivalent to grade 7 in the U.S. education system) were recruited as interviewees.

Among them, 14 participants were female and 10 were males. According to their

teacher, these participants come mainly from low to middle income families, and were acquainted with one another.

\section{Procedures}

Altogether 24 participants were recruited. They were divided into four groups, including two groups of seven girls each and two groups of five boys each. Written consent was obtained from the participants' parents and verbal consent was obtained from the participants before interviewing. The focus-group discussion sessions took from 35 to 65 minutes for each group. A female research assistant with a master's degree in communication acted as the moderator. The study was conducted in Mandarin, the official Chinese language.

The study consisted of two parts. In the first part, participants were asked to write down the food and beverages they usually consumed in addition to regular meals on a 
typical day in four particular time periods: morning, after lunch, afternoon (including after school), and after dinner. In the second part, an A3-size board with 10 snacks and beverages, including fruit, chocolate, digestive biscuits, soft drink, yogurt drink, ice cream, milk, chips, nuts, and cream biscuits were shown. These snacks were selected based on their popularity among Chinese children from a recent survey (Shanghai Consumer Protection Commission, 2014) and contained a mix of healthy and unhealthy items. Participants were asked to identify which of the snacks were healthy and which were unhealthy. An open-ended discussion session of basis for identification of healthy and unhealthy food, as well as factors influencing food choice began after these two activities.

\section{Instrument and data analysis}

Seven open-ended questions were designed (Appendix 1). While the board of snacks was shown as a visual prompt, the questions were about food items or food in general to capture participants' perspectives of food choices in a broader sense. Snacks were not specifically mentioned in the questions. The results of the first four questions are reported in this paper. The discussion sessions were audio recorded and later transcribed in Chinese. One of the authors read the transcripts several times to familiarize herself with the data. Constant comparative method was adopted to analyze and generate meaningful categories in order to explore and investigate the 
phenomenon systematically (Strauss, 1987). Based on the answers to the research questions, topic codes were created. The coder stopped the coding when new codes stopped appearing (Charmaz, 2006). The identified themes were examined by another author to minimize subjectivity in the analysis (Weber, 2004). The second reader received the identified theme and cross-checked against the transcripts for accuracy. Discrepancies, if any, were reconciled through discussion. Representative quotes were selected by the authors and translated into English. The transcripts indicated that there was not much interaction within the group. In most situations, participants responded to the questions posted by the facilitator. This may be because participants are unfamiliar with the focus group discussion setting.

The food and beverages consumed as snacks were categorized according to a study of children's and youths' food markets (Shanghai Consumer Protection Commission, 2014). Two categories, food purchased from street vendors and bottled tea, were added. (In cities across China, there are mobile food retailers on the streets that cook and sell food items such as pancakes and fried meat.)

\section{Results}

\section{Snacking behavior}

Participants' consumption of food and beverages on a typical day in addition to regular meals is summarized in Figure 1. Snacking behavior was common among 
participants. All 24 participants reported that they consumed two to 13 categories of food and beverages on a typical day. Fruit, milk/yogurt drink, and noodles/rice were the most frequently consumed snacks. Snacking behavior was quite evenly distributed throughout the day, being slightly more frequent in the morning than after dinner. It was interesting to note that milk, yogurt drink, and cake were not considered by participants as food consumed in a regular meal. Traditional Chinese breakfast items were rice porridge, steamed buns, and stuffed buns (Veeck et al., 2014). Milk and cakes were not regular breakfast items according to our understanding.

[Insert Figure 1 about here]

\section{Identification of healthy and unhealthy snacks}

Analysis of the interviews regarding how participants distinguish between healthy and unhealthy food generated three major themes: content and nutritional value of the food; whether the food is beneficial to physical and mental growth; and what parents and the media say about the food. The numbers of times these themes were brought up by the boys and girls are summarized in Figure 2 .

[Insert Figure 2 about here]

The most frequently mentioned criteria for identifying healthy or unhealthy food was content and nutritional value. Healthy foods were described as by participants foods that contain or provide protein, calcium, vitamins, water, (natural) sugar, fat, organic 
substances, and lactobacillus. Among these substances, protein and vitamins were most frequently mentioned. Similarly, unhealthy foods were described as foods that contain chemicals or artificial additives, artificial coloring, fat, salt, preservatives, and MSG. These were the main reasons why participants considered soft drinks and potato chips unhealthy. Four typical quotes read as follows:

"I think fruit is healthy, because it is rich in water and does not go through any artificial processing. It contains plenty of water, sugar and organic substances. It does not contain harmful elements. So I think fruit is a kind of healthy food.” (female)

"I choose fruit and yogurt. This is because fruit is nutritious. Yogurt contains lactobacillus. So, it is healthy." (male)

"Soft drinks are unhealthy. On their food labels numerous complicated, artificial chemical names are listed. I think they consist of chemicals and water only. There is no nutritional value in them." (female)

"I think potato chips are unhealthy because they contain too much fat." (female)

These four quotes showed that participants perceived a strong link between natural processing, natural ingredients, and healthiness. Based on the same grounds, chemical contents and additives were considered as unhealthy. Participants also perceived a strong link between unhealthy food and how the food was processed. Deep frying and preservation by salt or chemicals were perceived to make food unhealthy. Here is a typical quote:

"Potato chips are deep-fried . . . The deep-frying process releases toxic elements. Soft drinks contain chemical ingredients. They are harmful to our digestive system." (male) 
The second most frequently reported criterion for identifying healthy or unhealthy food was its effect on the physical health of young people. The emphasis was on whether the food would help them grow (benefit) or whether the food would make them fat (harm). Participants commented that they were at a critical stage of physical and mental growth, and that healthy food was essential to provide the energy and nutrition they needed for growth. Participants also mentioned that as students, they need nutritious foods to help them perform well academically. It is interesting to see the gender difference in the responses. Boys in the sample put much emphasis on physical growth while girls in the sample put more emphasis on body shape. Here are two typical quotes:

"Chips do not have the proteins or calcium that can help us grow. Chips only contain elements that are harmful to us. Soft drinks have too many carbohydrates. They are not suitable for our growth." (male)

"Unhealthy foods are those that can make people fat. These foods are usually rich in fat." (female)

The two quotes illustrated that participants were concerned about the immediate effects of food consumption on their bodies.

The third criterion for the identifying of healthy and unhealthy foods was reliance on word of mouth from parents, health advice found in the media, food labels, and the food pyramid. The following quote is representative: 
"I saw some surveys on the Internet of, for example, the 10 unhealthiest or healthiest foods. It is said that digestive biscuits are healthy." (female)

The participant quoted above relied on the media to tell her which foods were healthy and which were unhealthy.

Why adolescents consume healthy or unhealthy foods

The next two questions asked participants why some adolescents liked unhealthy foods or disliked healthy foods. The results are summarized in Figure 3. The top four factors affecting food choice were taste, image, convenience, and lack of concern. Taste was reported altogether 41 times as the reason for choosing unhealthy foods or not choosing healthy foods. Participants often described unhealthy foods as delicious, strongly flavored, or satisfying. On the other hand, participants tended to describe healthy foods as plain, mild, tasteless, not exciting, or even bitter. In general, participants preferred artificial flavor and strong taste to mild flavor. Furthermore, participants reported that artificial flavor and strong taste was addictive and would make consumers get used to it. They got so used to strongly flavored foods that they could no longer tolerate mildly flavored foods such as fruits and vegetables. Here are two typical quotes:

"In general, the taste of unhealthy food is pretty good. Once I begin to eat, I cannot stop. Healthy foods are all tasteless. Unhealthy foods are cheap and delicious, so I always eat them." (female)

"Healthy foods are all taste awful. The manufacturers want to sell more unhealthy 
foods. Therefore, they add a lot of additive to make the food tasty. These foods make us feel good. The taste is exactly what we want." (male)

In both quotes, the participants thought highly of the taste of unhealthy foods while regarded lowly the taste of healthy foods. They were also aware that the taste of unhealthy food was artificial and was manipulated to suit the consumer needs. It was also noticed that their views of taste represented subjective preconceptions, and were seldom supported by personal experience. Their judgments were less about the actual taste of healthy and unhealthy foods, and more their perceptions of the taste of such foods.

[Insert Figure 3 about here]

The second factor most frequently reported was image. Participants, boys in particular, found unhealthy foods exciting and interesting. Several participants commented that the marketing promotions of unhealthy foods were successful in making these foods trendy, funny, and eye-catching. On the other hand, healthy foods did not have attractive packaging, and appeared dull. Here are two typical quotes:

"The advertising promotion of unhealthy food is very good. For example, a candy is able to change the color of your tongue. There are always some new foods coming up. Young people will find them playful." (male)

"Consuming unhealthy foods such as soft drinks and chips is trendy now. The packaging is very pretty and attractive." (male) In both quotes, the participants appreciated the marketing efforts in promoting the unhealthy food. They also paid much attention to the visual presentation of the food. 
Because of the appealing marketing promotions, they came to associate unhealthy

foods with the appearance of modernity.

It was also interesting to find that participants often associate food choice with

self-identity or identify of a particular group of people in terms of geographic location,

age, or lifestyle. This is shown by these quotes:

"People in Hunan enjoy strong-flavored, salty, and spicy foods, and would rather choose them over fruit, which is comparatively mild-flavored." (female)

"We are in the rebellious period. Parents always ask us eat fruits and drink milk. We just don't want to follow them, so we dislike these foods." (male)

"Young people think nutritional fruits are outdated. ... I think they just follow the fashion of not pursuing a healthy life." (male)

In the first quote, the participant linked food choice to the culture of Hunan. In the second quote, the participant perceived himself as rebellious. Because of their self-identity, they would challenge authority and deliberately not choose food recommended by parents. In the third quote, the participant found that healthy food did not match with the image of young people.

The third and the fourth most frequently mentioned themes tied; these were convenience and lack of concern. Several participants reported inconvenience as the reason why adolescents disliked healthy food. They complained that consuming fruit, what with the necessary washing and peeling, was too troublesome. Preparing healthy food also involved a lot of time. On the other hand, unhealthy food was readily 
consumable, and was highly accessible. As one interviewee reported:

"People nowadays think that eating healthy food is time-wasting. They think it is too tedious. For example, if people want to eat porridge, they just buy a can of it at a supermarket." (female)

This quote showcases how the time involved in food preparation was a factor in influencing food consumption.

Lack of concern was also mentioned by participants as a factor affecting food choice.

They reported that young people are not particularly health conscious, and that nutrition contents of foods were not their major concern. Participants also believed that young people, without coming across immediate bad consequences of consuming unhealthy foods, didn't mind eating unhealthily. Here is an illustrative quote: "People think that they are healthy. As long as they are not sick, they did not see a need to consume healthy food." (male)

Besides these four factors, the other factors affecting food choices reported were peer influence and cost. Participants reported that unhealthy foods were more popular and cheaper. Here are two representative quotes:

"If you don't eat it while others are eating, you appear to be different from others. Others may say something [bad] behind your back." (female)

"All the foods sold by the street venders are junk. Healthy foods cost much more." (male)

\section{Discussion and conclusion}

A focus-group study was conducted to examine adolescents' snacking behavior and their perception of healthy and unhealthy foods. Similar to findings of previous 
studies, snacking behavior was popular among adolescents (Wang et al., 2012).

Findings of the current study indicated that interviewees consumed a mix of healthy and unhealthy snacks throughout different parts of the day. The high consumption of fruit after dinner suggests that our participants tended to stay home for dinner, where fruits are often served.

The identification of deep-fried foods and foods with preservatives or additives as unhealthy was similar to that among adolescents in Hong Kong (Chan et al., 2009a, b), as was the identification of natural foods and foods with calcium content (Chan et al., 2009a, b). Results also indicated that participants seldom used food labels to access information on nutrition content or calorie intake. This is probably because food labels are not readily available on packaging, especially for snacks bought from street vendors. The infrequent use of food labels among adolescents was consistent with what was found among Chinese adults (Liu et al., 2015).

While other studies found that adolescents often learn about guidelines related to safe and nutritious food from parents and grandparents (Veeck et al., 2014), the current study did not demonstrate strong parental guidance about choices of snacks. Only a few cited their parents as exerting significant influences to consume healthy foods such as milk and fruit. School teachers also did not play an important role in socializing children on snack choices. 
The importance of taste and image in food choices is demonstrated in this study.

While taste, nutrition, and weight control were found to be important guidelines for food choice in a previous study (Veeck et al., 2014), the importance of image in food choice is also worth noting. Even though the participants in this study reported the consumption of healthy snacks, they tended to perceive such snacks as boring, tasteless and non-delicious. They consumed fruit, milk, and yogurt drinks mainly because their parents and family members spent time preparing such items for them or coercing them to do so. Participants consistently expressed that they preferred the artificial flavor of unhealthy food to the mild flavor of healthy food. The findings supported a health-indulgence dilemma that traditional authorities were challenged by food marketers tempting adolescents towards indulgence (Bech-Larsen et al., 2010). This was probably why many of the interviewees desired and frequently consumed unhealthy, artificially flavored, but tasteful and convenient snacks. One interesting finding not revealed in previous studies is the perceived link between self-identity (or group-identity) and snack consumption. Several interviewees reverted to the provincial identity, or the stereotypical identity of a rebellious or fashionable youth to explain their preference for unhealthy food with strong taste. This suggests that the residential location of young consumers and the socially constructed meaning of adolescence can be factors that influence food culture. Further studies may consider 
adding these two variables. While concerns among girls over body figure are similar to those found by Veeck et al. (2014), the current study discovered similar concerns over physical growth among boys. Further study is needed to examine the specific aspects of growth that are of concern to them.

The current study suffers from the limitations of non-probability sampling, small sample size, confinement to interviewees from low and middle economic class, and use of recall behavior to understand snacking behavior of teenagers. Future study might profit from using quantitative survey or food diary methods to obtain data.

\section{Implications for parents, educators, and social marketers}

In order to promote healthy snacking behavior among Chinese adolescents, parents can take an active role by making healthy snacks more accessible at home and at schools. For example, parents can prepare fruit boxes for children to enjoy during the long school day. Parents should be aware that nagging their child to eat healthily is not an effective strategy. At the bottom-line, parents should pay attention the snacking patterns and the types of snacks their children are consuming.

Educators can teach adolescents how to read food labels. As convenience is important in food choice, schools could play a more important role by increasing the availability of healthy snacks on campus or even asserting influence on the street foods around the school vicinity. Social marketers need to be aware of the negative image of healthy 
food. Promotions that adopts rational appeals such as healthiness and nutritional values are unlikely to change the attitude and preference of adolescent consumers towards unhealthy snacking - ironically, the appeal itself may become the barrier to promoting healthy eating. On the one hand, some adolescents subconsciously directly associate the notion of "healthy snacks" with being boring and tasteless; on the other hand, such appeals may not be able to draw the attention of parents (who are also major buyers of snacks), as they generally consider their children's academic achievement to be more important than healthy eating. An example might be a mother allowing her son to have instant noodles late at night in order to keep him awake for study.

Instead, social marketing campaigns that put emphasis on the provision of healthy snacks with the ingredients for physical growth may be effective in drawing the attention of target parents and teenagers (especially boys). For public services advertisements promoting healthy eating, the fun and enjoyable part of consuming healthy snacks can become the key message. A mix-and-match consumption of healthy and unhealthy snacks (such as mixing yogurt drink or milk with biscuits or cake) can be demonstrated as a fun and preferred part of healthy snacking, without highlighting unhealthy snacks as undesirable - an approach that may generate the opposite effect among emotionally rebellious adolescents. 
Despite the fact that "taste" was reported as the main reason for liking unhealthy foods, taste as an indication of consumer preference (according to Bourdieu, 1984) is socially learned and constructed. In addition to parents being socializing agents, peer pressure also plays an important role. Some participants who find healthy snacks unappealing worry that such foods are outdated, and that their peers may say something bad behind their backs, indicating that the perception of consuming healthy food is equally important. Reframing healthy snacks as something fashionable, or cool, where health benefits are presented as merely a secondary advantage, might offer the opportunity for adolescents to relearn taste - to like eating healthy foods. Taste can be understood as a form of cultural capital. Acquiring a cultured palate that includes liking healthy foods can become a sign of being "high class". This may prove particularly effective, as in Changsha - named as a second-tier city of China and with its lower to middle income families - there is a drive toward upward social mobility. 


\section{References}

Bech-Larsen, T., Jensen, B.B. and Pedersen, S. (2010), “An exploration of adolescent snacking conventions and dilemmas", Young Consumers, Vol. 11 No. 4, pp. 253-263.

Bourdieu, P. (1984), Distinction: A Social Critique of the Judgement of Taste, trans. R. Nice, Routledge, London.

Brown, K., McIlveen, H. and Strugnell, C. (2000), "Nutritional awareness and food preferences of young consumers", Nutrition \& Food Science, Vol. 30 No. 2, pp. 230-35.

Buijzen, M., Schuurman, J. and Bomhof, E. (2008), “Associations between children's television advertising exposure and their food consumption patterns: a household diary-survey study", Appetite, Vol. 50 No. 2/3, pp. 231-39.

Chan, K., Prendergast, G., Grønhøj, A. and Bech-Larsen, T. (2009a), “Adolescents' perceptions of healthy eating and communication about healthy eating", Health Education, Vol. 109 No. 6, pp. 474-90.

Chan, K., Prendergast, G., Grønhøj, A. and Bech-Larsen, T. (2009b), "Communicating healthy eating to adolescents", Journal of Consumer Marketing, Vol. 26 No. 1, pp. 6-14.

Chen, Y. (2014), "Shanghainese children spend an average 10 Yuan on snacks each day; $70 \%$ of parents mistakenly believe that snacks are nutritious", Eastday.com, 30 May, available at: http://sh.eastday.com/m/20140529/u1a8117470.html (assessed 28 Oct 2015) [in Chinese].

Charmaz, K. (2006), Constructing Grounded Theory: A Practical Guide through Qualitative Analysis, Sage, London.

Croll, J.K., Neumark-Sztainer, D. and Story, M. (2001), "Healthy eating: what does it mean to adolescents?", Journal of Nutrition Education, Vol. 33 No. 4, pp. 193-98.

Du, S.F., Wang, H.J., Zhang, B., Zhai, F.Y. and Popkin, B.M. (2014), "China in the period of transition from scarcity and extensive undernutrition to emerging nutrition-related non-communicable diseases, 1949-1992”, Obesity Reviews, Vol. 15 Suppl. 1, pp. 8-15.

Economist, The (2012), "Food for thought", 15 December, available at: http://www.economist.com/news/special-report/21568064-food-companies-playambivalent-part-fight-against-flab-food-thought (accessed 27 February 2015).

EU SME Centre (2013), "The F\&B market in China", available at: http://www.ccilc.pt/sites/default/files/docs/mercado_dos_alimentos_e bebidas_e n_eusmecenter.pdf (accessed on 9 February 2015).

Fitzgerald, A., Heary, C., Nixon, E. and Kelly, C. (2010), "Factors influencing the food 
choices of Irish children and adolescents: a qualitative investigation", Health Promotion International, Vol. 25 No. 3, pp. 289-98.

Guo, Y. (2000), "Family relations: The generation gap at the table", Jing, J. (Ed.), Feeding China's Little Emperors: Food, Children, and Social Change, Stanford University Press, Stanford CA, pp. 94-113.

HKTDC (2014), "Changsha (Hunan) city information". available at: http://china-trade-research.hktdc.com/business-news/article/Fast-Facts/Changsha -Hunan-City-Information/ff/en/1/1X000000/1X09W9YB.htm (accessed 29 August 2014).

Kelly, B., Hebden, L., King, L., Xiao, Y., Yu, Y., He, G., Li, L., Zeng, L., Hadi, H., Karupaiah, T., Hoe, N.S., Noor, M.I., Yoon, J., Kim, H. (2014), “Children's exposure to food advertising on free-to-air television: an Asia-Pacific perspective", Health Promotion International, available at: http://heapro.oxfordjournals.org/content/early/2014/07/04/heapro.dau055.full.pdf +html (assessed 28 October 2015).

Kline, S. (2011), Globesity, Food Marketing and Family Lifestyles, Palgrave Macmillan, Basingstoke.

Larson, N. and Story, M. (2013), "A review of snacking patterns among children and adolescents: What are the implications of snacking for weight status?", Childhood Obesity, Vol. 9 No. 2, pp. 104-15.

Li, B., Adab, P. and Cheng, K. K. (2015), "The role of grandparents in childhood obesity in China - evidence from a mixed methods study", International Journal of Behavioral Nutrition and Physical Activity, Vol. 12 Article 91.

Liu, Z., Zhang, G. and Zhang, X. (2014), "Urban street foods in Shijiazhuang city, China: Current status, safety practices and risk mitigating strategies", Food Control, Vol. 41, pp. 212-18.

Marshall, D. (2010), Understanding Children as Consumers, Sage, London.

McGinnis, J.M., Gootman, J. and Kraak, V.I. (2006), Food Marketing to Children and Youth: Threat or Opportunity, The National Academies Press, Washington, DC.

Mullich, J. (2011), “China's 'second-tier' cities take off”, Wall Street Journal, available at: http://online.wsj.com/ad/article/chinaenergy-cities (accessed 8 July 2015).

Neuman, L. (2003), Social Research Methods: Qualitative and Quantitative Approaches, Allyn \& Bacon, Boston, MA.

Piernas, C. and Popkin, B.M. (2010), "Snacking increased among U.S. adults between 1977 and 2006", Journal of Nutrition, Vol. 140 No. 2, pp. 325-32.

Powell, L.M., Szczypka, G., Chaloupka, F.J. and Braunschweig, C.L. (2007), "Nutritional content of television food advertisements seen by children and 
adolescents in the United States", Pediatrics, Vol. 120 No. 3, pp. 576-83.

Shanghai Consumer Protection Commission (2014), "Shanghai Consumer Protection Commission releases Investigative Report on Snack Food and Drink Consumption Habits of Shanghainese Children (2014)", available at: http://www.cca.org.cn/xxgz/detail/15489.html (accessed 23 November 2015) [in Chinese].

Shepherd, J., Harden, A. Rees, R., Brunton, G., Garcia, J., Oliver, S. and Oakley, A. (2006), Young people and healthy eating: a systematic review of research on barriers and facilitators", Health Education Research, Vol. 21 No. 2, pp. 239-57.

Shi, X., Lien, N., Kumar, B.N. and Holmboe-Ottesen, G. (2005), "Socio-demographic differences in food habits and preferences of school adolescents in Jiangsu Province, China”, European Journal of Clinical Nutrition, Vol. 59 No. 12, pp. $1439-48$.

Silverman, D. (2005), Doing Qualitative Research (2nd Edition), Sage, London.

Stevenson, C., Doherty, G., Barnett, J., Muldoon, O.T. and Trew, K. (2007), "Adolescents' views of food and eating: identifying barriers to healthy eating", Journal of Adolescence, Vol. 30 No. 3, pp. 417-34.

Strauss, A.L. (1987), Qualitative Analysis for Social Scientists, Cambridge University Press, Cambridge, MA.

Sun, C. (2014), "Fast-food chains reinvent themselves as Chinese tastes evolve", available at:

http://www.scmp.com/business/china-business/article/1556072/fast-food-chainsreinvent-themselves-chinese-tastes-evolve (accessed 9 February 2015).

Taillie, L.S., Afeiche, M.C., Eldridge, A.L. and Popkin, B.M. (2015), "Increased snacking and eating occasions are associated with higher energy intake among Mexican children aged 2-13 years", Journal of Nutrition, available at: http://jn.nutrition.org/content/early/2015/09/16/jn.115.213165.full.pdf+html (assessed 28 October 2015).

The American Chamber of Commerce in Shanghai (n.d.), "What is meant by first tier, second-tier, and third-tier cities?", available at:

http://sme.amcham-shanghai.org/en/faq/what-meant-first-tier-second-tier-and-thi rd-tier-cities (accessed 8 July 2015).

The World Factbook (2015). Retrieved from https://www.cia.gov/library/publications/resources/the-world-factbook/ (assessed 23 November 2015)

U.S. Department of Health and Human Services (1988), The Surgeon General's Report on Nutrition and Health, U.S. Government Printing Office, Washington, DC. 
Veeck, A., Grace Yu, F., Yu, H., Veeck, G. and W. Gentry, J. (2014), "Influences on food choices of urban Chinese teenagers", Young Consumers, Vol. 15 No. 4, pp. 296-311.

Waller, C.E., Du, S. and Popkin, B.M. (2003), "Patterns of overweight, inactivity, and snacking in Chinese children" Obesity Research, Vol. 11 No. 8, pp. 957-61.

Wang, Z., Zhai, F., Du, S. and Popkin, B.M. (2008), "Dynamic shifts in Chinese eating behaviors", Asia Pacific Journal of Clinical Nutrition, Vol. 17 No. 1, pp. 123-30.

Wang, Z., Zhai, F., Zhang, B. and Popkin, B.M. (2012), “Trends in Chinese snacking behaviors and patterns and the social-demographic role between 1991 and 2009”, Asia Pacific Journal of Clinical Nutrition, Vol. 22 No. 2, pp. 253-62.

Weber, R. (2004), "The rhetoric of positivism versus interpretivism: A personal view", MIS Quarterly, Vol. 28 No. 1, 429-449.

Yang, J., Yao, S., Zhu, X., Zhang, C., Ling, Y., Abela, J.R., Esseling, P.G. and McWhinnie, C. (2010), "The impact of stress on depressive symptoms is moderated by social support in Chinese adolescents with subthreshold depression: A multi-wave longitudinal study", Journal of Affective Disorders, Vol. 127 No. 1, pp. 113-21.

Young, B. (2003), “Does food advertising make children obese?", Young Consumers, Vol. 4 No. 3, pp.19-26.

Yu, D., Zhang, B., Zhao, L. and Wang, H. (2008), "Snacks consumption in Chinese children and adolescents at the ages of 3-17 years", Journal of Hygiene Research, Vol. 37 No. 6, pp. 710-13.

Zhai, F.Y., Du, S.F., Wang, Z.H., Zhang, J.G., Du, W.W. and Popkin, B.M. (2014), "Dynamics of the Chinese diet and the role of urbanicity, 1991-2011", Obesity Reviews, Vol. 15 Suppl. 1, pp. 16-26.

Zhang, B., Zhai, F.Y., Du, S.F. and Popkin, B.M. (2014), "The China Health and Nutrition Survey, 1989-2011”, Obesity Reviews, Vol. 15 Suppl. 1, pp. 2-7. 


\section{Appendix 1 English translation of the questions asked in the focus-group sessions}

1. Please tell me why some of the food items on the display board are healthy.

2. Please tell me why some of the food items on the display board are unhealthy.

3. Why do some adolescents like unhealthy food?

4. Why do some adolescents dislike healthy food?

$5^{*}$. Can you recall a food advertisement that you like the most? Please tell us what is in the ad and why you like it.

$6^{*}$. In your daily life, have you come across any kind of food promotion?

$7 *$. Suppose you are a government officer and you are designing ways to encourage young people to eat more fruit. Here are some of the suggested ways. Please tell us which one(s) is effective and why?

a) Producing an interesting TV commercial to publicize the benefits of eating fruit;

b) Designing an online game to generate good impressions about fruit;

c) Sending text messages or emails to remind young people to eat fruit;

d) Offering some interesting free gifts to encourage people to buy fruit;

e) Recruiting celebrities appealing to youths to publicize the benefits of eating fruit;

f) Sponsoring sports activities for young people to generate good impressions about eating fruit.

* Not reported in this paper 
Figure 1. Consumption of snack at different day parts

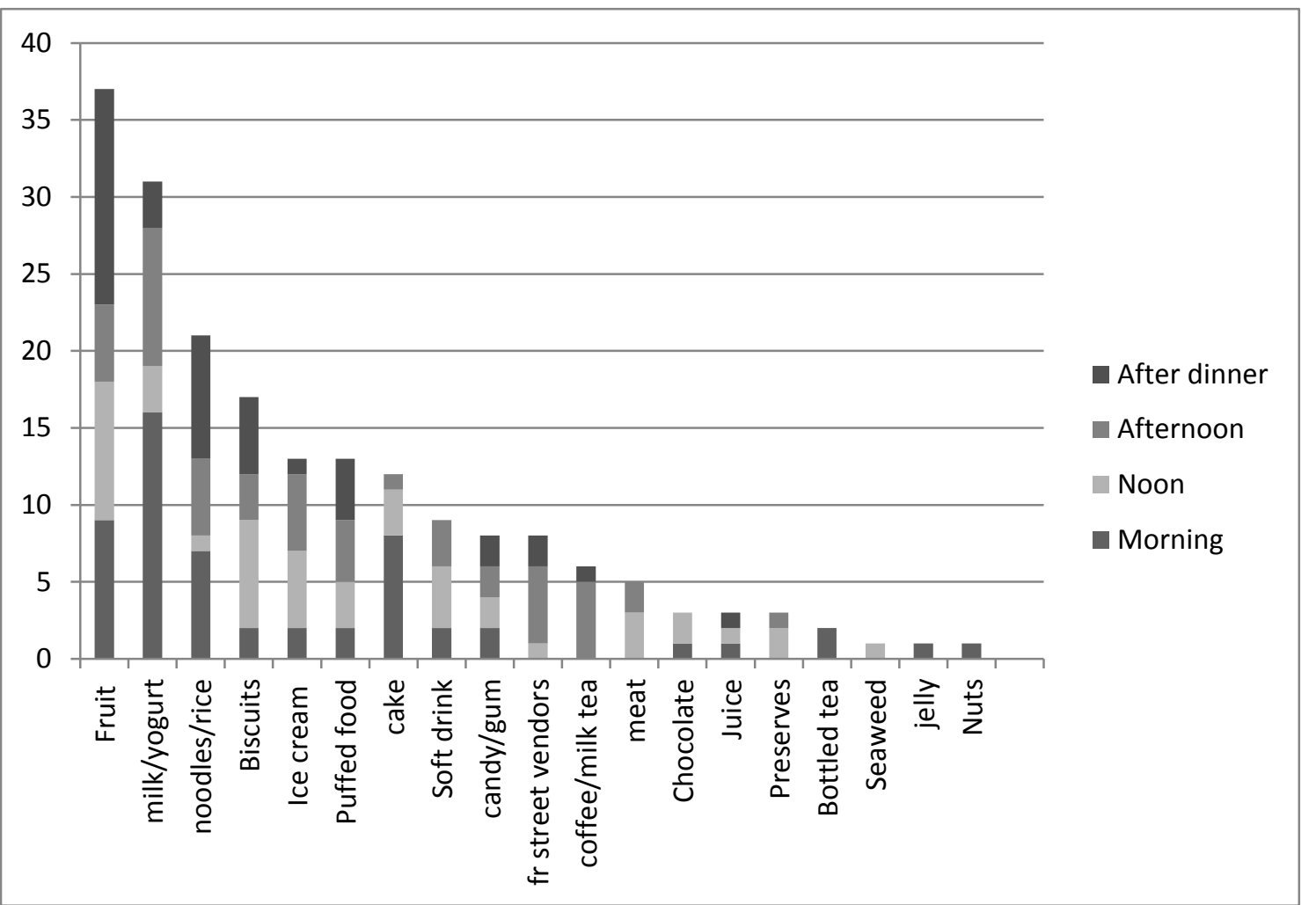


Figure 2. Basis for identifying healthy and unhealthy foods by sex of participants

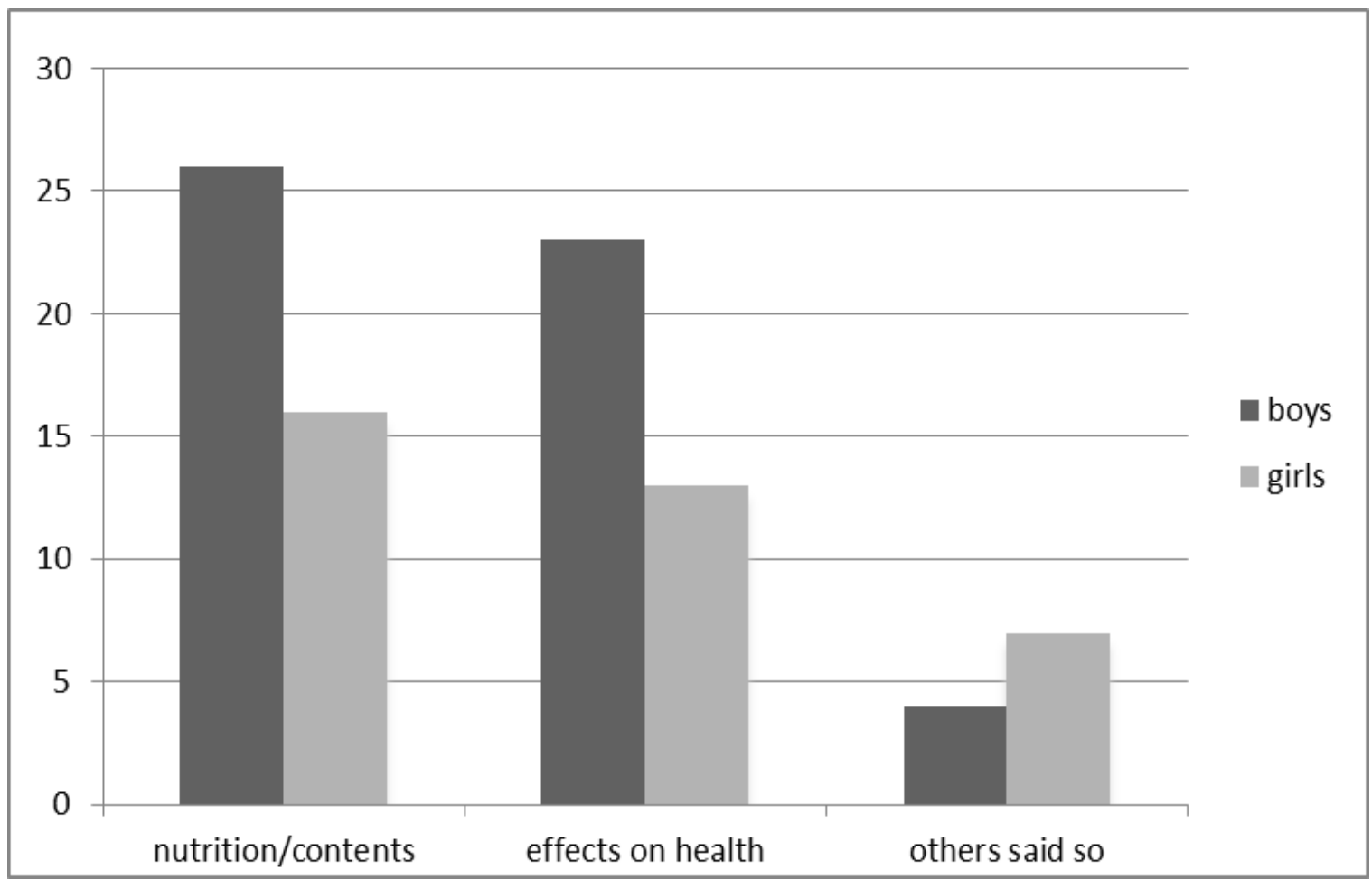


Figure 3. Factors affecting food choice by sex of participants

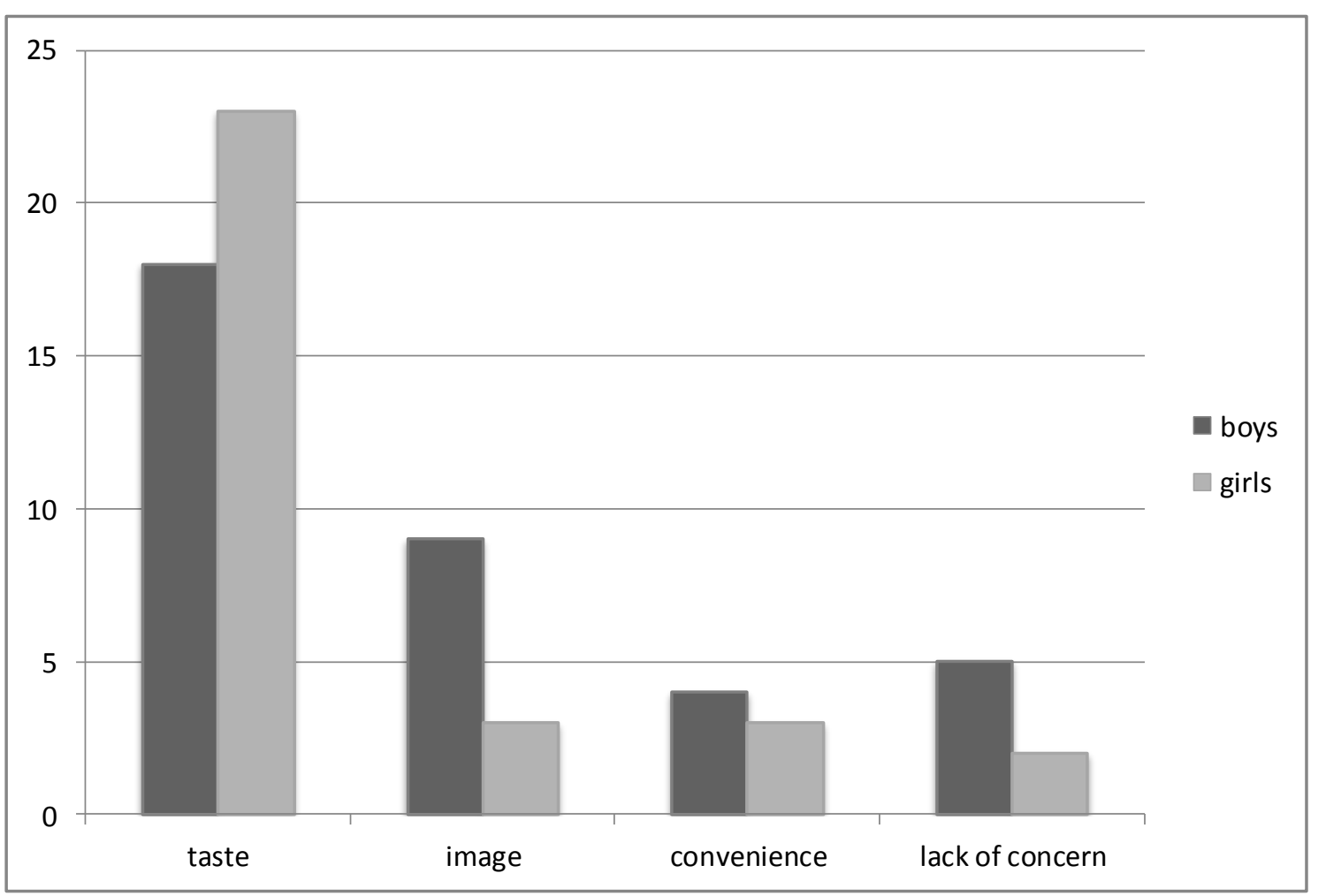

